\title{
Propagating-path uniformly scanned light sheet excitation microscopy for isotropic volumetric imaging of large specimens
}

\author{
Junyu Ping \\ Fang Zhao \\ Jun Nie \\ Tingting Yu \\ Dan Zhu \\ Mugen Liu \\ Peng Fei
}




\title{
Propagating-path uniformly scanned light sheet excitation microscopy for isotropic volumetric imaging of large specimens
}

\author{
Junyu Ping, ${ }^{\mathrm{a}, \dagger}$ Fang Zhao, ${ }^{\mathrm{a}, \dagger}$ Jun Nie, ${ }^{\mathrm{a}, \dagger}$ Tingting $\mathrm{Yu},{ }^{\mathrm{b}}$ Dan Zhu, ${ }^{\mathrm{b}}$ Mugen Liu, ${ }^{\mathrm{c}}$ and Peng Fei ${ }^{\mathrm{a}, \mathrm{b}, \mathrm{d}, \star}$ \\ ${ }^{a}$ Huazhong University of Science and Technology, School of Optical and Electronic Information, Wuhan, China \\ ${ }^{b}$ Huazhong University of Science and Technology, Britton Chance Center for Biomedical Photonics, Wuhan National Laboratory for \\ Optoelectronics, Wuhan, China \\ 'Huazhong University of Science and Technology, College of Life Science and Technology, Wuhan, China \\ dHuazhong University of Science and Technology, Shenzhen Research Institute, Shenzhen, China
}

\begin{abstract}
We demonstrate a propagating-path uniformly scanned light sheet excitation (PULSE) microscopy based on the oscillation of voice coil motor that can rapidly drive a thin light sheet along its propagation direction. By synchronizing the rolling shutter of a camera with the motion of laser sheet, we can obtain a uniform planeilluminated image far beyond the confocal range of Gaussian beam. A stable 1.7- $\mu \mathrm{m}$ optical sectioning under a $3.3 \mathrm{~mm} \times 3.3 \mathrm{~mm}$ wide field of view (FOV) has been achieved for up to $20 \mathrm{~Hz}$ volumetric imaging of large biological specimens. PULSE method transforms the extent of plane illumination from one intrinsically limited by the short confocal range ( $\mu \mathrm{m}$ scale) to one defined by the motor oscillation range ( $\mathrm{mm}$ scale). Compared to the conventional Gaussian light sheet imaging, our method greatly mitigates the compromise of axial resolution and successfully extends the FOV over 100 times. We demonstrate the applications of PULSE method by rapidly imaging cleared mouse spinal cord and live zebrafish larva at isotropic subcellular resolution. $\odot$ The Authors. Published by SPIE under a Creative Commons Attribution 4.0 Unported License. Distribution or reproduction of this work in whole or in part requires full attribution of the original publication, including its DOI. [DOI: 10.1117/1.JBO.24.8.086501]
\end{abstract}

Keywords: light-sheet microscopy; biomedical optics; imaging systems; optical design; isotropic resolution; three-dimensional reconstruction.

Paper 190185LR received Jun. 4, 2019; accepted for publication Jul. 15, 2019; published online Aug. 5, 2019.

In the past decade, light sheet fluorescence microscopy has become an emerging technique for three-dimensional (3-D) imaging of thick samples at high speed and low photo toxicity. ${ }^{1-9}$ For a conventional focused Gaussian sheet, a conflict always exists between the beam confocal range and its axial extent, thereby causing a trade-off between high axial resolution and large field of view (FOV). One possible approach is to iteratively illuminate the sample and stitch these tiles together. ${ }^{10,11}$ But the mechanical stitching greatly limits the throughput. Recently, light sheet combined with nondiffracting beams, such as Airy beam $^{6,12}$ or Bessel beam, ${ }^{3,13,14}$ can generate a thin light sheet over a long distance, which mitigates the conflict of axial resolution and FOV. However, compared to Gaussian beam that focuses most of photons into the beam waist, these nondiffracting beams have more dispersed energy at the side lobes. Though a few techniques such as confocal virtual slit ${ }^{15,16}$ have been developed to eliminate the fluorescence emission by the side lobes' excitation, the specimen still receives the photon burden from the side lodes. More complicated modalities, such as two-photon Bessel light sheet ${ }^{3,17,18}$ and lattice light sheet, ${ }^{14}$ are reported to physically suppress the side lodes, but these systems are in a more complex format and at a higher cost.

An alternative approach is to sweep a thin Gaussian light sheet along the propagation direction to dynamically extend the confocal range of illumination. Some special elements, such as a mirror based on a piezo stage or voice coil motor (VCM), ${ }^{19,20}$

\footnotetext{
*Address all correspondence to Peng Fei, E-mail: feipeng@ hust.edu.cn

†equal contributing author
}

electrical tunable lens (ETL), ${ }^{21-24}$ or tunable acoustic gradient lens ${ }^{25,26}$ are combined with confocal virtual slit to achieve this goal. For example, with using an ASLM the extended confocal depth can reach $162 \mu \mathrm{m}$ with an axial resolution of $390 \mathrm{~nm} .{ }^{19}$ The use of ETL, which has a larger adjustable range than a piezo scanner, can further extend the confocal range to $\sim 1 \mathrm{~mm}$ with $\sim 4$ to $6 \mu \mathrm{m}$ resolution. ${ }^{23}$ However, for these methods, the beam scanning is performed before the illumination objective. The convergence angle of incident light entering the rear pupil of illuminating objective varies during the light-sheet sweeping. Thus, it is difficult for these methods to maintain a uniform light-sheet thickness at very large scanning range of several millimeters. $^{24}$

Here, we propose a propagating-path uniformly scanned light sheet excitation (PULSE) method via directly oscillating the illumination objective by a VCM. By synchronization of the VCM-driven light-sheet motion and the confocal slit of an sCMOS camera, we can achieve a uniform light sheet sectioning of $1.7 \mu \mathrm{m}$ over a large FOV of $3.3 \mathrm{~mm} \times 3.3 \mathrm{~mm}$ in single acquired frame. At the same time, the VCM can push a speed limit of 20 frames per second. We successfully apply this technique to the volumetric imaging of mouse spinal cord and live zebrafish larva, proving isotropic subcellular resolution across entire samples.

In conventional LSFM, the incident Gaussian beam is focused in one dimension and forms a thin laser sheet. The axial extent of laser sheet, which usually determines the system axial resolution, can be defined as the diameter of beam waist $d$ (FWHM value). According to the Gaussian beam propagation theory, ${ }^{27} d$ can be calculated as 
$d=\sqrt{2 \ln 2} \frac{\lambda f}{\pi \omega_{0}}$,

where $f$ is the focal length, $\lambda$ is the excitation wavelength, and $\omega_{0}$ is the radius of the incident beam. Meanwhile, the confocal range, which is appropriate for imaging, can be expressed as

$l=\frac{\pi d^{2}}{\ln 2 \lambda}=\frac{4 \lambda f^{2}}{\ln 2 \pi \omega_{0}^{2}}$.

It is obvious that as the thickness of laser sheet goes smaller (higher axial resolution), the illumination range (FOV) decreases drastically. As a reference point, a $2-\mu$ m-thick lightsheet only yields $\sim 38-\mu \mathrm{m}$ confocal range, which is only suited for imaging single cell. Therefore, imaging millimeter-size large samples at single-cell axial resolution becomes a choice dilemma of either tens of microns low axial resolution or slow stepwise mosaic stitching.

In our PULSE setup, the incident beam was line-focused first by a cylindrical lens (Thorlabs, LJ1695RM) and then formed the illuminating laser sheet through a long working-distance objective (Mitutoyo, $10 \times / 0.28 \mathrm{NA}$ ). The objective was mounted steadily on a VCM (TMEC0050-025-000) for high-speed oscillation under a millimeter-scale large amplitude, inducing an oscillating light-sheet covering the entire FOV [Fig. 1(a)]. In the static mode, the thin light sheet measured $\sim 1.7 \mu$ m only covers a small imaging area $\sim 28 \mu \mathrm{m}$. Under $4 \times$ objective detection, the quality of acquired image degrades seriously beyond this range [Fig. 1(h)]. Through controlling the rolling shutter of an SCMOS camera (Hamamatsu. ORCA-Flash 4.0 V2), we formed a linear array that contains 15 to 20 rows of active pixels sweeping at the sensor plane and tightly synchronized it with the VCM-driven movement of the Gaussian sheet waist [Figs. 1(b), 1(e), and 1(f)] using a customized Labview program. This dynamic synchronization mode transformed the extent of plane illumination from one limited by the short confocal range to one defined by the motor oscillation range. The formed confocal electronic slit further blocked the fluorescence resides excited by the diverged

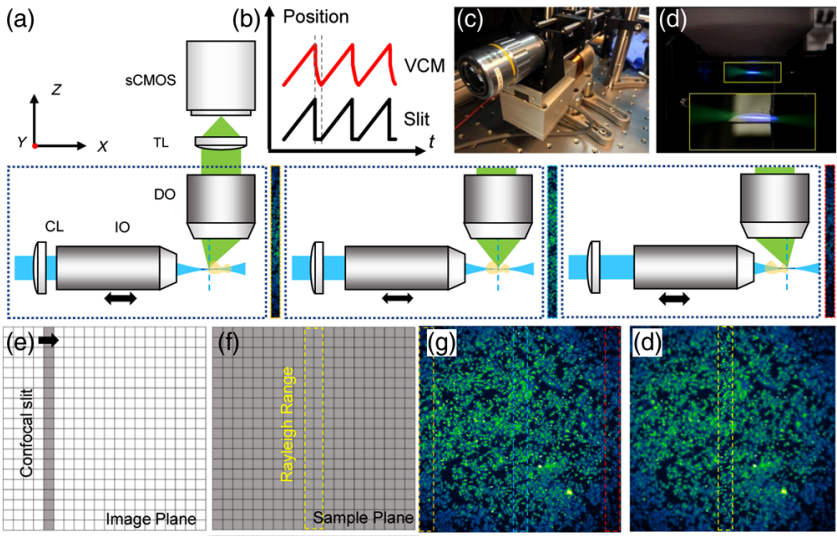

Fig. 1 (a) Schematic of the propagational light sheet scan using VCM. The confocal range of the laser sheet rapidly moving along the $x$ direction. (b) Control timing diagram of the VCM motion and rolling shutter of the camera. (c) Device photo of VCM mounted with objective. (d) Through the synchronization, a virtual confocal slit is formed to exclusively collect the fluorescence excited by the Gaussian sheet waist (Video 1), as shown in (e) and compared to conventional static light-sheet geometry in (f). (g), (h) The plane images acquired under PULSE mode and conventional mode, respectively. (Video 1, MPEG, 5.47 MB [URL: https://doi.org/10.1117/1.JBO.24.8.086501.1]). laser out of the confocal range. Thus, we can obtain sharp planeexcited fluorescence signals in single wide-FOV frame recorded by $4 \times$ objective [Fig. 1(g)]. Then through a $z$-scan controlled by another independent actuator (Thorlabs, Z825B), a 3-D stack can be obtained at a high speed of tens of PULSE frames per second. Video 1 [Fig. 1(d)] shows the process of light-sheet moving and synchronizing with the camera. The photograph of VCM mounted with objective is shown in Fig. 1(c).

Compared to propagational scanning using ETL, ${ }^{22}$ scanning the laser sheet by directly driving the illuminating lens can generate a more uniform optical sectioning when the sample is across a large range of several millimeters. We used ZEMAX software to simulate the profiles of the focused Gaussian sheet generated by PULSE and ETL methods (EL-10-30-VIS-LD, Optotune, Switzerland). PULSE method maintained a very uniform laser sheet thickness of $\sim 1.7 \mu \mathrm{m}$ after the illuminating objective traveling a $3.3-\mathrm{mm}$ distance along propagation direction [Fig. 2(a), bottom row and Fig. 2(b), orange line]. In contrast, the thickness of light sheet would varies over 35\% (from 1.7 to $2.3 \mu \mathrm{m}$ ) via using the ETL to shift the focal point of Gaussian sheet with the same $3.3 \mathrm{~mm}$ distance [Fig. 2(a), top row and blue line]. This nonuniform plane illumination would result suboptimal contrast and resolution while imaging large samples.

We used fluorescent beads ( $\sim 500 \mathrm{~nm}$, Lumisphere) as point source to experimentally characterize our system. We first evaluated the excitation efficiency of PULSE via comparing it with conventional static plane illumination set-ups. A 10X/0.4 detection objective was used for collecting the fluorescence signals excited by the laser sheets of different thickness. The narrow confocal range of $1.7 \mu \mathrm{m}$ sheet was measured around $28 \mu \mathrm{m}$ in static mode [Fig. 3(a)]. The confocal virtual slit of the camera was accordingly set to 40-pixel lines ( $26 \mu \mathrm{m})$ under PULSE mode, with rolling speed over 10 full frames per seconds synchronized with the VCM objective scanning. The acquired plane image of the beads is shown in Fig. 3(b). Due to the more intensive energy condensed at the beam waist, the thinner light sheet enables stronger fluorescence emission and higher signal contrast as compared to the thicker light sheets [ 3 and $8 \mu \mathrm{m}$ in Figs. 3(c) and 3(d)]. Meanwhile, comparing the PULSE and static excitation, the application of the confocal virtual slit does not affect the emission intensity, generating uniform and strong signals across the entire frame [Figs. 3(e) and 3(f)].

Then, a lower magnification of $4 \times$ detection was used to compare the effective imaging FOV under PULSE and conventional static models. With obtaining the $z$-stacks using both
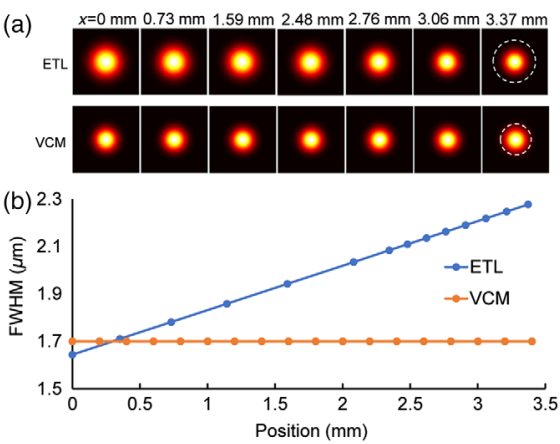

Fig. 2 (a) The beam profile at different propagation position adjusted by ETL (top row) and VCM (bottom row). (b) The thickness of light sheet at different focusing position by ETL (blue line) and VCM (orange line). 

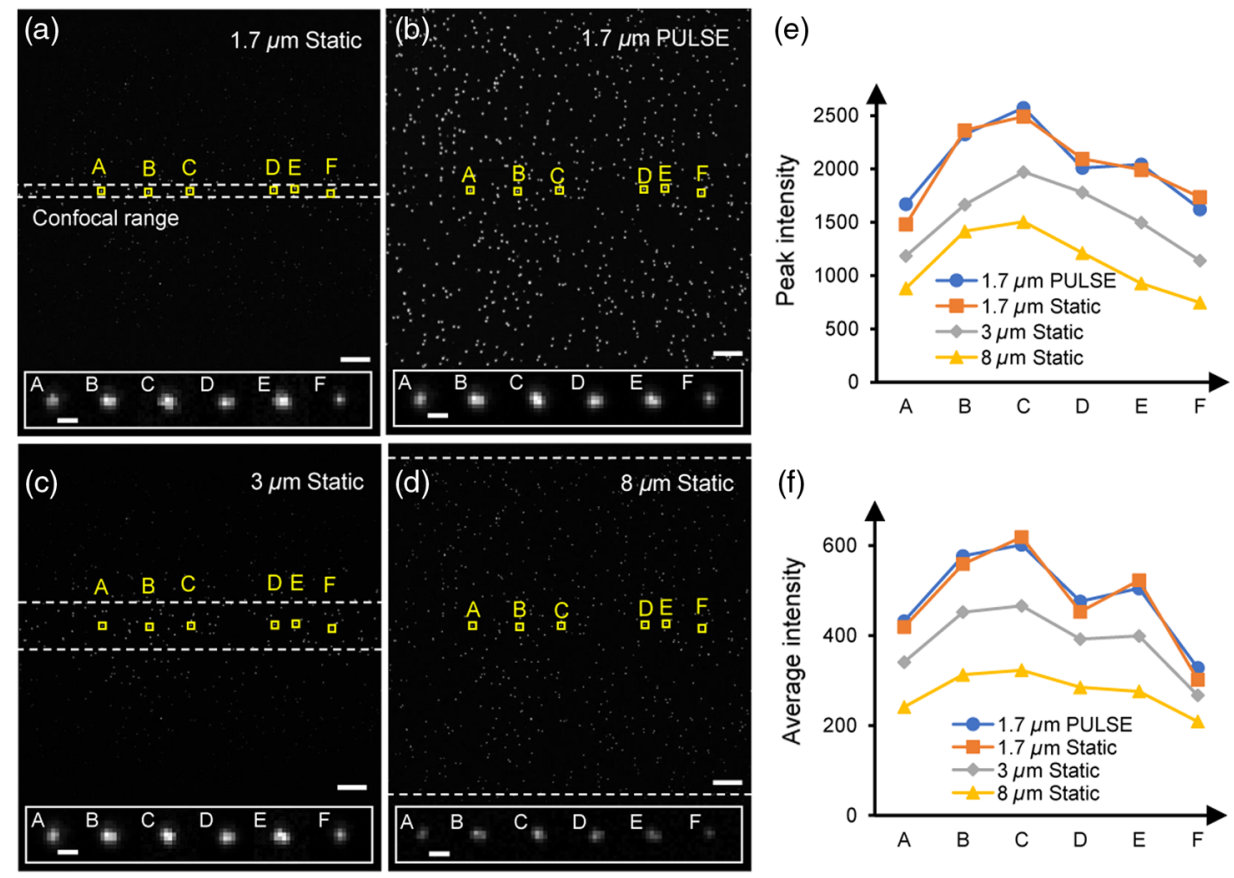

Fig. 3 (a)-(d) The signals distribution of fluorescent beads acquired using 1.7- $\mu \mathrm{m}$ static sheet, 1.7- $\mu \mathrm{m}$ PULSE, 3- $\mu \mathrm{m}$ static sheet, and 8- $\mu \mathrm{m}$ static sheet, respectively. (e), (f) Comparison of peak and averaged intensities of six selected signals shown in (a)-(d). Scale bars: $50 \mu \mathrm{m}$ in (a)-(d) and $3 \mu \mathrm{m}$ in insets.
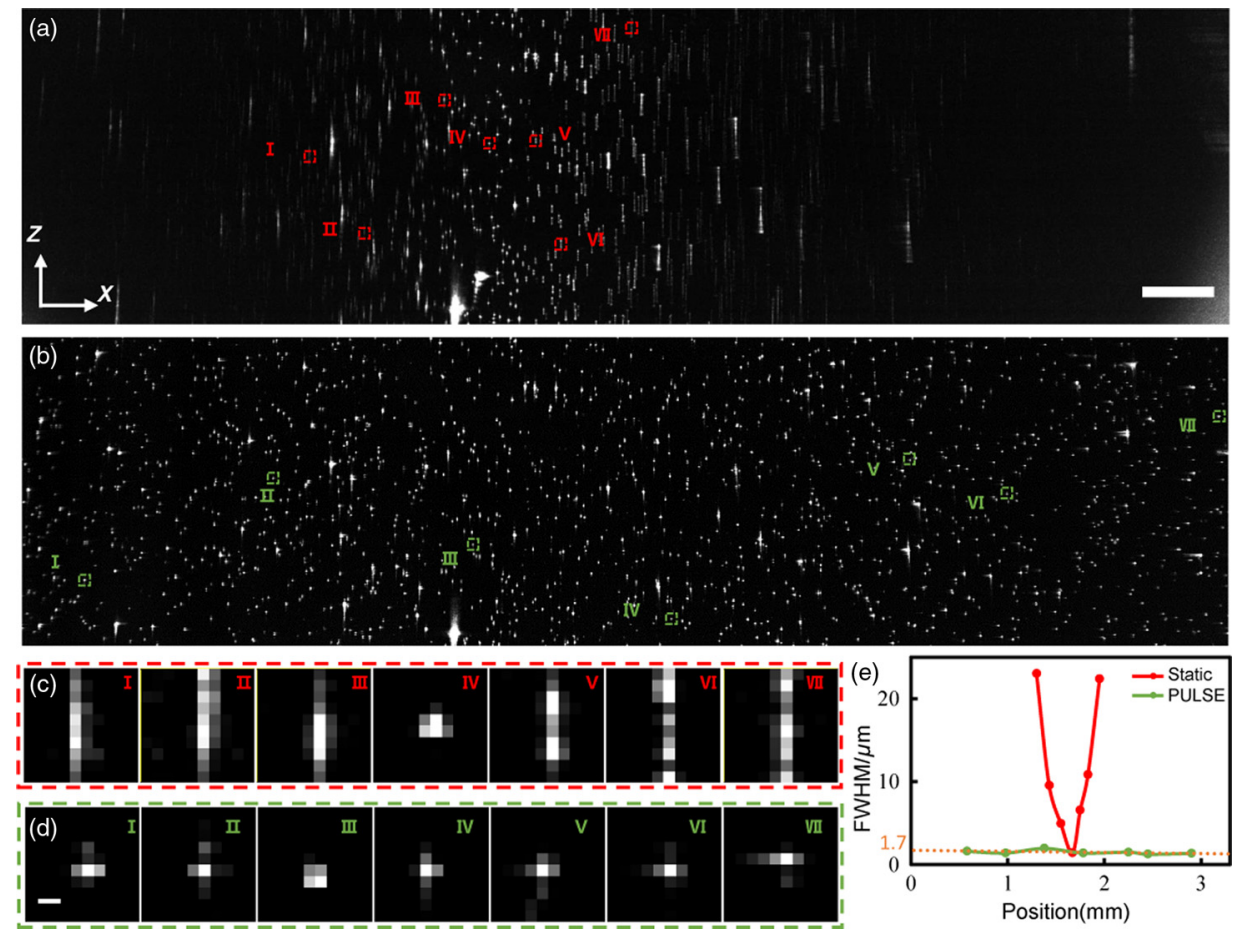

Fig. 4 (a), (b) The reconstructed $x z$ planes of fluorescent beads imaged by a 1.7- $\mu \mathrm{m}$ thin light sheet under conventional and PULSE modes. (c), (d) Vignette high-resolution views of seven selected beads at different $x$ positions in (a) and (b). (e) The variations of axial intensity profiles of the selected beads in (c) and (d). Scale bars: $200 \mu \mathrm{m}$ in (a), (b) and $5 \mu \mathrm{m}$ in (c), (d).

methods, seven reconstructed beads at different $x$ positions were accordingly extracted from both setups to analyze their axial performance [Figs. 4(c) and 4(d)]. The measured axial extents of the beads from conventional light sheet shows over 10-times divergence (1.7 to $\sim 20 \mu \mathrm{m}$ ) within a $500-\mu \mathrm{m}$ short propagation.
This is in significant contrast with very sharp-and-uniform optical sectioning of PULSE (1.7 to $1.8 \mu \mathrm{m}$ ) across entire 3.3-mm FOV. Our method obviously mitigates the compromise of axial resolution while greatly extends the effective FOV over 100 times. 
We demonstrated PULSE's ability via three-dimensionally imaging fluorescence-tagged mouse spinal cord (thy1-GFP) and zebrafish larva ( $T g$ flk1: mCherry). The PULSE setup was identical with that described above, and a $10 \times / 0.4$ apochromatic objective lens was used to collect fluorescent signals at adequate lateral resolution. Figure 5(a) showed the 3-D reconstruction of spinal cord by PULSE microscopy. The center of confocal range is around $x=700 \mu \mathrm{m}$. Three $y z$ planes with $200-\mu \mathrm{m}$ interval in propagation direction $(x)$ were extracted and shown in Fig. 5(b). The same $y z$ sample planes from the 3 -D reconstructions by 1.7 and $8 \mu \mathrm{m}$ static laser sheet were shown in Figs. 5(c) and 5(d) as comparison. Due to the tradeoff between the axial resolution and the FOV, $8-\mu$ m laser sheet has a better over performance across the large scale [Figs. 5(d2), 5(d3)] while the 1.7- $\mu \mathrm{m}$ sheet shows thinner optical sectioning ability at the narrow beam waist [Fig. 5(c1)]. In consistence with the PSF test, static excitation mode caused either signal deterioration out of small confocal range [Figs. 5(c2), 5(c3)] or insufficient overall resolution
[Fig. 5(d)] while PULSE showed high-and-uniform axial resolution capable of discerning neural fibers in both planes. In Fig. 5(e), the 3-D visualization of the vascular system in live zebrafish larva also verified an isotropic resolution has been achieved in all transverse $(x y)$, coronal $(x z)$, and sagittal $(y z)$ views.

In summary, we have reported the PULSE microscopy method, which is based on the synchronization of VCMdriven light-sheet oscillation and the rolling virtual slit of the sCMOS camera. PULSE method greatly extends the FOV of conventional Gaussian light-sheet imaging by two orders while maintaining a thin-and-uniform optical sectioning. As a demonstration, PULSE can readily provide a gigavoxel image of millimeter-thick samples, such as mouse spine cord and zebrafish larval, with isotropic subcellular resolution achieved at a speed up to $20 \mathrm{fps}$. The highest frame rate of PULSE is currently limited by the load of VCM and its large travel range. We believe this speed limitation could be overcome via further optimizing

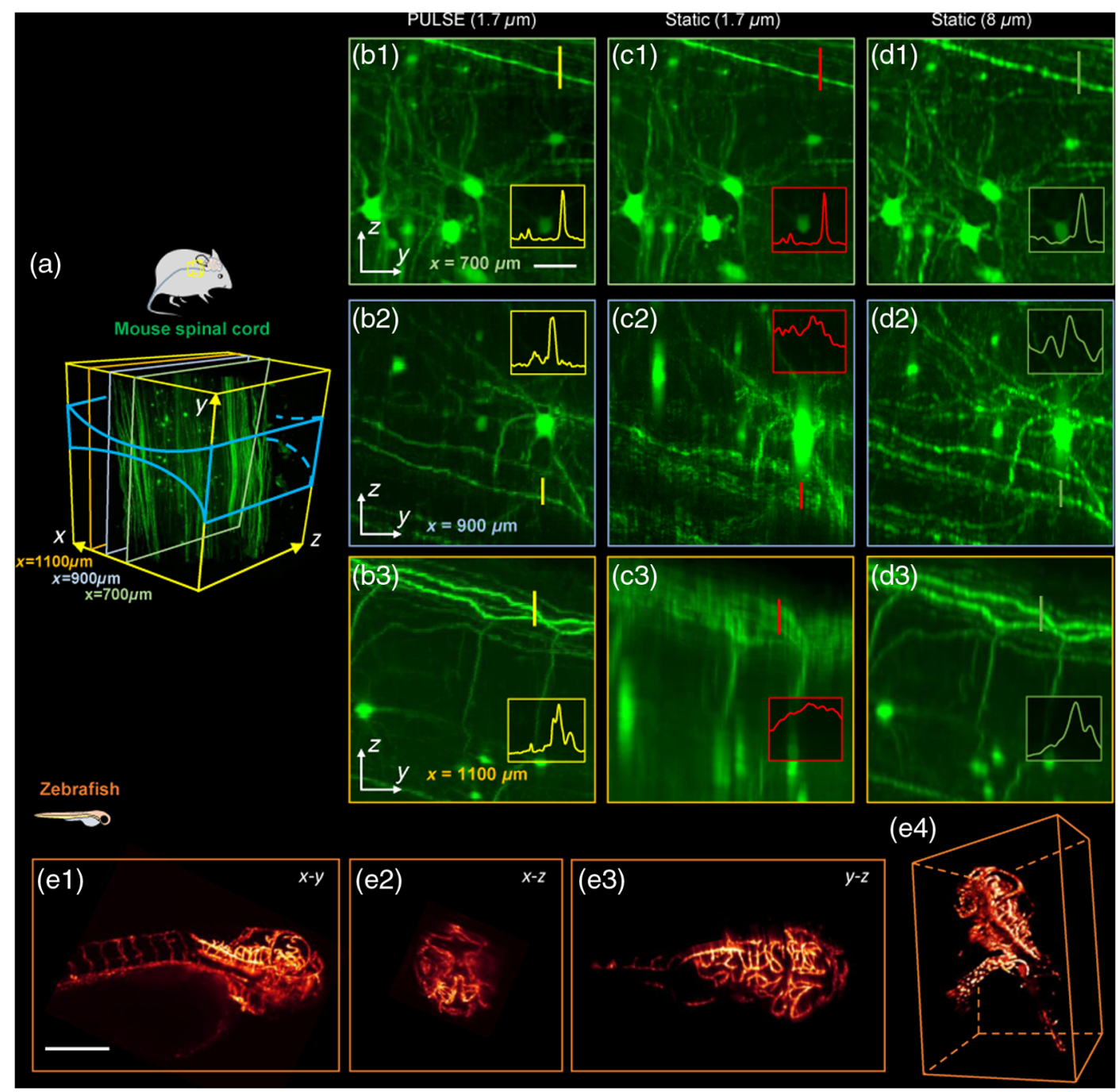

Fig. 5 (a) The 3-D reconstruction of cleared mouse spinal cord with neurons labeled by GFP (thy1-GFP). (b) Three reconstructed $y z$ planes with $200 \mu \mathrm{m}$ interval in the propagation direction $(x)$, showing the uniform isotropic resolution spanning a wide view. These results are further compared with the images by 1.7 and $8 \mu \mathrm{m}$ static sheets, as shown in (c), (d), respectively. Insets in (b)-(d), line intensity profiles of the neuron fibers, indicating the different resolving power of three methods. (e) PULSE imaging of a blood vessel-tagged zebrafish larval ( $T g$ flk1: mCherry). (e1)-(e3), The $x y, x z$, and $y z$ views of the anterior part of the zebrafish. (e4) The 3-D volume rendering of the zebrafish. Scale bars: $50 \mu \mathrm{m}$ in (b)-(d) and $100 \mu \mathrm{m}$ in (e). 
the design. By choosing VCM with a better actuator, improving the mechanical design, and integration of vibration isolator, it is possible to obtain a higher scanning frequency well matched to the frame rate of the camera. The advancements of simplified setup and thin-and-uniform illumination across ultralarge range render the PULSE method a promising tool for a variety of biomedical applications, such as embryo development, tissue histology, pathology, and neuron science research, in which high-resolution cellular profiling at whole-tissue scale is highly desired.

\section{Disclosures}

The authors have no relevant financial interests in the manuscript.

\section{Acknowledgments}

The authors acknowledge Yusha Li and Jiayi Tu for providing the mouse and zebrafish samples and funding support from National Key R\&D Program of China (P.F., 2017YFA0700500), the National Natural Science Foundation of China (21874052), Research Program of Shenzhen (P.F., JCYJ20160429182424047), and 1000 Youth Talents Plan of China (P.F.).

\section{References}

1. J. Huisken et al., "Optical sectioning deep inside live embryos by selective plane illumination microscopy," Science 305, 1007-1009 (2004).

2. P. J. Keller et al., "Reconstruction of zebrafish early embryonic development by scanned light sheet microscopy," Science 322, 1065-1069 (2008).

3. T. A. Planchon et al., "Rapid three-dimensional isotropic imaging of living cells using Bessel beam plane illumination," Nat. Methods 8, 417-423 (2011).

4. U. Krzic et al., "Multiview light-sheet microscope for rapid in toto imaging," Nat. Methods 9, 730-733 (2012).

5. M. B. Ahrens et al., "Whole-brain functional imaging at cellular resolution using light-sheet microscopy," Nat. Methods 10, 413-420 (2013).

6. T. Vettenburg et al., "Light-sheet microscopy using an Airy beam," Nat. Methods 11, 541-544 (2014).

7. S. Wolf et al., "Whole-brain functional imaging with two-photon lightsheet microscopy," Nat. Methods 12, 379-380 (2015).

8. J. Lee et al., "4-Dimensional light-sheet microscopy to elucidate shear stress modulation of cardiac trabeculation," J. Clin Invest. 126, 1679-1690 (2016).
9. R. M. Power and J. Huisken, "A guide to light-sheet fluorescence microscopy for multiscale imaging," Nat. Methods 14, 360-373 (2017).

10. L. Gao, "Extend the field of view of selective plan illumination microscopy by tiling the excitation light sheet," Opt. Express 23, 6102-6111 (2015).

11. Q. Fu et al., "Imaging multicellular specimens with real-time optimized tiling light-sheet selective plane illumination microscopy," Nat. Commun. 7, 11088 (2016).

12. G. Siviloglou et al., "Observation of accelerating Airy beams," Phys. Rev. Lett. 99, 213901 (2007).

13. L. Gao et al., "Noninvasive imaging beyond the diffraction limit of 3D dynamics in thickly fluorescent specimens," Cell 151, 1370-1385 (2012).

14. B.-C. Chen et al., "Lattice light-sheet microscopy: imaging molecules to embryos at high spatiotemporal resolution," Science 346, 1257998 (2014).

15. E. Baumgart and U. Kubitscheck, "Scanned light sheet microscopy with confocal slit detection," Opt. Express 20, 21805-21814 (2012).

16. F. O. Fahrbach and A. Rohrbach, "Propagation stability of selfreconstructing Bessel beams enables contrast-enhanced imaging in thick media," Nat. Commun. 3, 632 (2012).

17. F. O. Fahrbach et al., "Light-sheet microscopy in thick media using scanned Bessel beams and two-photon fluorescence excitation," Opt. Express 21, 13824-13839 (2013).

18. M. Zhao et al., "Cellular imaging of deep organ using two-photon Bessel light-sheet nonlinear structured illumination microscopy," Biomed. Opt. Express 5, 1296-1308 (2014).

19. K. M. Dean et al., "Deconvolution-free subcellular imaging with axially swept light sheet microscopy," Biophys. J. 108, 2807-2815 (2015).

20. T. Chakraborty et al., "Light-sheet microscopy with isotropic, submicron resolution and solvent-independent large-scale imaging," bioRxiv (preprint): 605493 (2019).

21. A. K. Chmielewski et al., "Fast imaging of live organisms with sculpted light sheets," Sci. Rep. 5, 9385 (2015).

22. P. N. Hedde and E. Gratton, "Selective plane illumination microscopy with a light sheet of uniform thickness formed by an electrically tunable lens," Microsc. Res. Tech. 81, 924-928 (2018).

23. B. Migliori et al., "Light sheet theta microscopy for rapid highresolution imaging of large biological samples," BMC Biol. 16, 57 (2018).

24. F. F. Voigt et al., "The mesoSPIM initiative: open-source light-sheet mesoscopes for imaging in cleared tissue," bioRxiv (preprint): 577122 (2019).

25. K. M. Dean and R. Fiolka, "Uniform and scalable light-sheets generated by extended focusing," Opt. Express 22, 26141-26152 (2014).

26. W. Zong et al., "Large-field high-resolution two-photon digital scanned light-sheet microscopy," Cell Res. 25, 254-257 (2015).

27. M. Born and E. Wolf, Principles of Optics, 7th ed., Cambridge University Press, Cambridge (1999).

Biographies of the authors are not available. 